\title{
The acute effects of resistant starch on appetite and satiety in overweight individuals
}

\author{
N. M. Al-Mana and M. D. Robertson \\ University of Surrey, Guildford, Surrey, GU2 7WG, UK
}

\begin{abstract}
Numerous studies have linked higher intakes of dietary fibre to the management of body weight ${ }^{(1,2)}$. The effect of fibre on weight may be mediated by different mechanisms; such as changes in blood glucose and insulin concentrations, reduction in food intake, slowing gastric emptying time and changes to anorexic hormone release after fibre consumption. Resistant starch (RS) is a form of dietary fibre which can be consumed at higher doses than similar fibres. RS, therefore, may have an important role in food intake, as we have previously demonstrated in normal weight individuals ${ }^{(3)}$. The aim of the present study was to investigate the short-term effects of RS on appetite and satiety in overweight/obese subjects and to investigate the effects of the RS on glucagon-like peptide-1(GLP-1) release.

Ten healthy male obese subjects (aged $18-32$ years, BMI $28-37 \mathrm{~kg} / \mathrm{m}^{2}$ ) participated in this single blind, randomized, crossover study. Subjects were required to consume a test breakfast and lunch, containing either $48 \mathrm{~g}$ of an RS (HAM-RS2) supplement or an energy and carbohydrate matched placebo split over two meals. Subjects recorded ratings of hunger, satiety, fullness and prospective food consumption using visual analogue scales (VAS) every $30 \mathrm{~min}$ for $7 \mathrm{~h}$. Postprandial blood glucose, insulin and GLP-1 responses were also measured. Energy intakes from an ad libitum dinner and for the remainder of the day were assessed.

The $48 \mathrm{~g}$ dose of RS had no significant impact on qualitative feelings of satiety or hunger. The RS supplement significantly reduced the energy intake at the ad libitum meal compared with PL(4465 (SEM 700) kJ for RS versus 5319 (SEM 805) kJ respectively, ( $p=0.017$ ). However, there were no significant effect for RS over 24 hour compared to PL $(p=0.508)$. RS ingestion led to significantly higher plasma glucose levels $(p=0.004)$ compared to PL, while the postprandial insulin response were not significant different. There were also a trend for higher c-peptide concentrations after RS intake $(p=0.089)$, but no significant differences in either fasting or postprandial levels of GLP-1 $(p=0.580)$. These results suggest that RS may indeed be beneficial in reducing acute meal intake in overweight/obese individuals.
\end{abstract}

1. Kendall CWC, Esfahani A \& Jenkins DJA (2010) The link between dietary fibre and human health. Food Hydrocolloids 24, 42-48.

2. Ludwig DS, Pereira MA, Kroenke CH et al. (1999) Dietary fiber, weight gain, and cardiovascular disease risk factors in young adults. JAMA 282, $1539-46$.

3. Bodinham CL, Frost GS \& Robertson MD (2010) Acute ingestion of resistant starch reduces food intake in healthy adults. Br J Nutr 103, 917-922. 